\title{
Trivium
}

Revue franco-allemande de sciences humaines et sociales - Deutsch-französische Zeitschrift für Geistesund Sozialwissenschaften

$3 \mid 2009$

Droits subjectifs et droits de l'homme

\section{La théorie de l'ordre et les droits naturels}

\section{Niklas Luhmann}

Traducteur : Olivier Mannoni

\section{OpenEdition}

Journals

Édition électronique

URL : http://journals.openedition.org/trivium/3277

DOI : $10.4000 /$ trivium.3277

ISSN : 1963-1820

Éditeur

Les éditions de la Maison des sciences de l'Homme

Référence électronique

Niklas Luhmann, "La théorie de l'ordre et les droits naturels », Trivium [En ligne], 3 | 2009, mis en ligne le 15 avril 2009, consulté le 10 décembre 2020. URL : http://journals.openedition.org/trivium/3277 ;

DOI : https://doi.org/10.4000/trivium.3277

Ce document a été généré automatiquement le 10 décembre 2020.

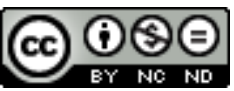

Les contenus des la revue Trivium sont mis à disposition selon les termes de la Licence Creative Commons Attribution - Pas d'Utilisation Commerciale - Pas de Modification 4.0 International. 


\title{
La théorie de l'ordre et les droits naturels
}

\author{
Niklas Luhmann
}

Traduction : Olivier Mannoni

\section{NOTE DE L'ÉDITEUR}

Nous remercions chaleureusement la Loewenklau Gesellschaft de nous avoir accordé le droit de traduire cet article de Niklas Luhmann.

\section{I.}

La théorie des droits subjectifs, telle que nous la connaissons couramment, s'est tellement éloignée de sa forme originelle qu'il est difficile de reconstituer les intentions qui l'ont d'abord guidée. Depuis la seconde moitié du xvIII siècle, les droits subjectifs ne sont plus conçus que comme les barrières d'un ordre de droit objectif, déterminé par le législateur. On sait aujourd'hui que la distinction entre « subjectif » et « objectif » n'est pas d'un grand secours pour ce qui concerne ce concept. On a cependant conservé la catégorie du droit subjectif. Compte tenu de la positivisation de l'ensemble du droit et du droit de disposition du législateur sur les constitutions, elle était trop importante pour pouvoir céder aux réserves théoriques. Il faut tout de même bien qu'existe une quelconque limite à l'arbitraire législatif, et l'idée de droits subjectifs pouvait suggérer qu'il fallait chercher ces barrières dans la valeur propre de l'homme. Elles ont donc été exprimées et inscrites dans les constitutions sous la forme des droits fondamentaux. Du point de vue pratique, cela ne signifie toutefois qu'une seule chose: pourvoir les constitutions de capteurs spécifiques, capables de percevoir la mutation sociale. En un mot: Lorsque les situations changent, n'oubliez pas l'homme! Personne ne voudra affirmer, et a fortiori imposer le contraire, et pourtant ce programme invite à se demander si l'on a ainsi suffisamment appréhendé le problème et la valeur 
fonctionnelle de la figure de la théorie. Quand on lit des textes du XVII siècle, on est confronté à une vision totalement différente de la situation théorique. Sa structure est bien plus axée sur la théorie de l'ordre, elle est déterminée par de tout autres problématiques, et elle n'a pas du tout été conçue, dans un premier temps, dans la perspective de la protection ou comme un simple programme complémentaire à la positivisationdu droit. Il pourrait donc s'avérer profitable de revenir sur le concept des natural rights - ne fût-ce que pour se demander qui a évacué les problèmes importants de l'époque, et où ils l'ont été.

II.

2 Aucun ordre juridique ne peut admettre que l'on demande s'il a été institué dans le droit ou dans le non droit. C'est un problème pour écrivains ${ }^{1}$, éventuellement pour sociologues, mais avant tout pour théoriciens du système. Dans le cadre du système du droit, le refoulement de la question concernant le droit de faire usage de la contrainte au nom du droit, du droit de faire usage du droit et du droit de traiter continuellement des questions encore non tranchées dans le droit à tous les niveaux du système, cesse très rapidement d'être productif. Il faut partir du caractère obligatoire du droit en le considérant comme une donnée initiale ${ }^{2}$. Si l'on applique la distinction entre le droit et le non-droit à cette distinction même, on aboutit ou bien à des paradoxes, ou bien à des impossibilités logique de trancher; car lorsqu'on dit que le sens de cette distinction réside dans cette distinction même ou dans une bifurcation correspondante des opérations du système, le droit d'introduire la distinction est le droit d'introduire le non-droit. Dans la logique la plus abstraite dont on dispose pour traiter ce problème, le calcul de George Spencer Brown, l'application de la distinction à la distinction n'est donc pas prévue, ou bien elle est assimilée à l'extinction de la distinction ${ }^{3}$. Le codage du système par le code du système lui-même devrait, selon les recherches Jorge Luis Borges, forcer le système à oublier précisément cette opération, et le système ne pourrait tolérer des textes qui mentionnent malgré tout cette opération qu'à la condition que ces textes soient pour leur partchiffrés ${ }^{4}$.

3 Il se trouve que la catégorie des "droits naturels" semble avoir, à l'origine, été appliquée précisémentà ce problème. Elle constitue une contribution au chiffrage d'un texte qui se situait à proximité de ce paradoxe et ne pouvait donc pas dire simplement ce que ce texte signifiait. Ce sont forcément des motifs historiquement perceptibles qui, vers le milieu du XvII e siècle, ont donné l'occasion de s'y essayer d'une manière nouvelle par rapport aux efforts produits antérieurement. Mais cela, on ne pourra l'établir que si l'on comprend plus précisément comment les théories correspondantes sont élaborées au contact du problème. Il va de soi que des problèmes doivent être évacués à cette occasion, compte tenu de la nature du problème fondamental. Reste tout de même à savoir comment cela se produit et pourquoi cela se produit de la manière dont cela se produit. Car toute déparadoxalisation du paradoxe est frappée du stigmate de la contingence.

III.

4 C'est bien entendu Thomas Hobbes qu'il faut citer en premier. Chacun s'y attendra. Il a cependant écrit une théorie trop excentrique pour nos fins, et en son temps trop peu 
consensuelle. Nous préférons donc nous en tenir à un autre texte, le Ductor Dubitantium ${ }^{5}$ de Jeremy Taylor. Taylor (1613-1667), un contemporain de Hobbes, a pris, sur des bases résolument théologiques, une position très voisine sur la question du droit naturel. Son point de départ est la distinction entre natural rights et natural law. Les droits naturels sont des droits dépourvus de toute valeur d'ordre, à rapportés l'homme en faisant abstraction de la société. "The right of nature is a perfect and universal liberty to do whatsoever can secure or please $m e^{6}$." Sa nature donne à l'homme des "appetites » en conséquence, qui s'autorisent eux-mêmes, dans une certaine mesure, leur activation. Ce qu'ils ne peuvent pas garantir, c'est la réciprocité des obligations. Dans l'état de nature, le droit est un droit sans obligations, raison pour laquelle il ne suppose aucune sorte de loi. On pourrait dire qu'il s'agit d'un droit seulement à demi réalisé, né du fait que l'individu prend ses propres appetites comme fil directeur et les définit, pour luimême, comme la norme ${ }^{7}$. C'est seulement lorsqu'on veut en outre créer des devoirs et harmoniser mutuellement droits et devoirs, que l'on a besoin de lois naturelles. Or cela est nécessaire pour que s'instaure un droit mis en ordre. Le droit est par suite considéré comme une limitation de soi-même. La constitution du droit est en conséquence un processus à deux paliers, le deuxième palier de la loi naturelle limitant le premier. On ne crée pas de devoirs correspondant à chaque appétit, ni à chaque droit naturel. Un processus de sélection est mis en place entre les uns et les autres. Hobbes le considère comme la sélection de la sélection: l'institution de la domination politique. Pour Taylor, c'est encore Dieu qui reste responsable, y compris des lois naturelles.

5 C'est seulement au deuxième palier, celui de la loi naturelle, que la contrainte devient nécessaire, car il s'agit désormais de garantir la sélection contingente de l'harmonisation des droits et des devoirs contre toute action contraire. Penalty and coercion sont introduits "for without these there is no law ${ }^{8}$ ". Le gain issu de la combinaison doit donc se payer par les institutions supplémentaires que sont la peine et son exécution. Dans l'ancien droit naturel, la raison aidait encore à avancer un peu sur ce point. Hooker, notamment, avait aussi fondé sur la raison la validité du droit naturel. Dans cette constellation désormais transformée, dans la justification en deux paliers du droit par le droit et la loi, cela n'est plus possible, car les droits, eux aussi, sont à leur manière voulus par Dieu et rationnels. La raison se cantonne à une capacité subjective à reconnaître le droit et laisse au pouvoir coercitif le soin de faire valoir les lois. Le continuum de rationalité qui avait porté la connaissance et ses objets, l'agir et ses normes, se déchire. «For reason can demonstrate, and it can persuade and invite, but not compel any thing but assent, not obedience, and therefor it is no law ${ }^{9} »$ ?

6 La sélection opérée par la loi naturelle qui pourvoit les droits des devoirscorrespondants, est attribuée à Dieu, et la validité des lois a son dernier motif dans la volonté de Dieu. On reprend le volontarisme théologique d'un Ockham, mais dans ce nouveau contexte il débouche sur la question de savoir pourquoi Dieu choisit ce procédé à deux paliers alors qu'il aurait tout aussi bien pu créer tout de suite des droits intégrés à l'ordre juridique. Tout cela ne ressemble-t-il pas beaucoup à la correction après coup d'une erreur dans la création? Dans un premier temps, le traitement de cette question déçoit. Il ne fait que répéter la théorie : Dieu a créé l'homme libre, et cela signifie (!) doté de désirs ${ }^{10}$, et il a ensuite limité son champ d'action par des lois naturelles que l'homme peut discerner. Seules les lois permettent de différencier le droit et le non-droit. C'est par conséquent quelque chose comme une logique de la 
liberté qui rend nécessaire ce procédé à deux paliers, et pour le même motif, la raison reste réduite à la connaissance.

7 Le point central de l'argumentation de Taylor réside dans la justification et la défense du concept volontariste de la loi ${ }^{11}$ avec ses conséquences, la dépendance à l'égard de l'acte de volonté de Dieu, la relativité historique et liée au réel. La loi, comme le dit Taylor, est "superinduced upon human nature», mais malgré tout nature, en tant que nature voulue par Dieu.

8 Le paradoxe est donc au bout du compte absorbé par la théologie. Dieu ne peut pas commettre d'injustice, pas même en introduisant la différence entre droit et non droit. "Indeed God cannot do an unjust thing, because whatsoever He wills or does is therefore juste because He wills and does $i^{12}$. » Ou bien, en se référant explicitement à Ockham : «Nullus actus nisi quatenus a Deo prohibitus est, et qui non possit fieri bonus si a Deo praecipiatur, et $e$ converso $^{13} »$. Mais ce n'est que le point de vue interne, ou encore l'autodescription d'un univers constitué sur le mode religieux. Pour son observation interne, on peut chiffrer le paradoxe dans le concept de Dieu et ainsi lui ôter son caractère paradoxal. Mais un observateur externe pourrait très bien retrouver le paradoxe, il pourrait par exemple se demander si Dieu serait capable de donner à une pierre un poids tel que lui-même ne soit plus capable de la soulever ${ }^{14}$. Le discours théologique se justifie lui-même, mais ainsi il justifie trop, et il reste à se demander pourquoi l'on choisit la procédure à deux paliers, right - law, si, au bout du compte, les droits originels doivent forcément être éliminés, ou de nouveau approuvés par la loi ${ }^{15}$.

9 La réponse ne va pas sans détours. Le concept volontariste de loi force à rompre avec le présupposé d'un droit naturel homogène, éternel et uniforme. On ne doit pas penser que la qualité de l'auteur puisse être limitée par la qualité de son produit ${ }^{16}$. La supposition, dans un premier temps tellement plausible, qu'il existe un répertoire minimal de préceptes dotés d'une validité universelle, ceux qui caractérisent le monde de la morale naturelle, est repoussée dans une polémique contre un passage fameux d'Ulpien (Ius naturale est, quod natura omnia animalia docuit, D. 1.1.1.3) ${ }^{17}$. Une telle uniformité ne correspond pas selon lui à l'intention de Dieu, raison pour laquelle il régule le droit naturel sous la forme des lois. "The reason is, because that which is natural is one, but these laws admit variety; and among wise nations in several cases have and have not obligation ${ }^{18}$ ".

10 Ici, le sens de la procédure en deux paliers devient tout d'un coup visible : elle crée un espace de jeu pour une plus grande diversité, pour une plus grande complexité. Lié par une constellation initiale déjà conçue comme normative et par un continuum de rationalité qui fonde sous la forme du dictatum rectae rationis la validité et la connaissance sur une dimension, l'ordre ne peut pas se développer pour atteindre une complexité suffisante (et la vision élargie du monde qui est celle des temps modernes montre tout à fait clairement que le monde, tel qu'il est, ne peut pas être conçu ainsi). Raison pour laquelle la prétention normative de l'ancien droit naturel, qui avait été encore une fois formulée de manière exemplaire dans les Laws of Ecclesiastical Polity de Richard Hooker, a été remplacée par la distinction entre rights et law. Dans le cadre de cette distinction, le point de départ peut être déplacé vers les droits naturels et appréhendé sous forme négative. Les droits ne sont pas encore un ordre, ils ont besoin d'un ordre. Ils constituent un potentiel de pulsions naturelles (appetites) qui peut être réalisé par le biais d'une limitation. Seul le détour par un " excès » et la répression qui s'y rattache permet à l'ordre de devenir complexe. Et dans le même temps, le processus 
de répression devient lui-même transparent d'un point de vue fonctionnel et dirigeable par adaptation. En parlant et en agissant dans le temps historique, Dieu adapte les lois naturelles aux situations données par la nature, mais on passerait à côté de sa volonté si l'on voulait déjà interpréter comme des lois les situations elles-mêmes dans leurs fondements naturels.

\section{IV.}

Le chiffrage religieux du paradoxe épargne à la théorie l'obligation d'en traiter ailleurs ${ }^{19}$. Mais la plausibilité de ce genre de tentatives est déjà sur le déclin, et sous son patronage, on prépare déjà la succession, même si c'est dans un premier temps sous une forme seulement rudimentaire. Elle se retrouve dans les « contre-différenciations " d'un nouveau genre qui remettent en question l'ancien ordre hiérarchique (celui des ordres, mais aussi celui des sources du droit, des genres littéraires, des disciplines scientifiques) ${ }^{20}$. Au XVIII ${ }^{\mathrm{e}}$ siècle, elles porteront le nom de nature et civilisation, ou de légalité et moralité. Elles sont préparées chez Taylor, aussi bien quand il insiste sur les natural rights que lorsqu'il insiste sur la contrainte comme élément essentiel de la natural law.

Ce qui frappe avant tout, dans le paradigme des deux paliers, c'est le fait que le point de départ de la genèse est transposé du droit vers les droits, auxquels manquent tous les éléments essentiels du droit. Ici intervient sûrement aussi, vu sous l'angle de la politique des idées, un travail de sape de la sémantique politique par les mouvements radicaux des levelers ou des diggers qui, au cours des deux décennies précédant la parution du Ductor Dubitantium, avaient réclamé l'égalité des droits politiques et celle des droits économiques. On peut dire à présent : « d'accord, mais sans les devoirs qui les accompagnent!» On peut ainsi à la fois reconnaître ces revendications et les ignorer. Et l'on peut proposer ce qui suit : quiconque veut vivre avec ses natural rights dans un ordre social et obtenir qu'ils soient reconnus doit se plier aux conditions que Dieu a prévues pour cela (ou, chez Hobbes : aux conditions prévues par le pacte).

Mais ce n'est qu'une vision superficielle, historico-génétique, de ce constat. Nous en ferons, au sixième paragraphe de ce texte, une exploitation sous l'angle de la théorie de l'évolution. Ce qui nous intéresse dans le présent contexte, c'est la forme sémantique par laquelle le paradoxe du droit peut être représenté dans le système. Elle tient au codage dans les termes des droits et des devoirs, qui prendra ensuite une signification centrale pour toute la théorie du droit naturel au xvIII ${ }^{e}$ siècle. L'ancienne unité des droits et des devoirs dans le concept de ius se change en différence ${ }^{21}$. Dans un premier temps, les droits sont appliqués sans qu'on leur adjoigne de devoirs. Cela signifie que, de la même manière qu'en Dieu, on ne peut pas encore parler, dans leur cas, d'une différence entre droit et non-droit. Ils désignent seulement l'absence de véritable codage du droit en droit et non-droit et permettent ainsi de réfléchir, dans une sorte d'expérimentation intellectuelle, à l'utilité qu'il y aurait à disposer d'un code de ce type. Les droits naturels représentent dès lors le paradoxe. Ils conceptualisent plusieurs niveaux dans un même concept, ils sont une "tangled hierarchy", dans le sens où l'entend Hofstadter ${ }^{22}$. Ce sont des droits, mais s'ils sont tels, ce n'est pas par différence avec le non-droit: ce sont des droits par différence avec des devoirs. Si l'on veut parvenir au droit à partir de ces droits, on découvre nécessairement qu'en user relevait du non-droit. Quiconque insiste pour que ces droits soient réalisés se voit répondre 
qu'il est nécessaire pour cela d'y renoncer. Le paradoxe est chiffré par le type de relation entre deux distinctions, celle du droit et du non-droit, et celle des droits et des devoirs. Et Borges a parfaitement raison : dans ce jardin de senderos que se bifurcan, il est nécessaire de passer le temps sous silence : le temps dont on a besoin pour passer des droits (en excluant les devoirs) au droit (par différence avec le non-droit). Il est vrai qu'on ne peut pas dire simplement que Dieu a d'abord créé les droits naturels, puis rien du tout pendant longtemps, et pour finir, peu à peu, le droit naturel destiné à les corriger. Pour Hobbes aussi, cette nécessité de passer le temps sous silence s'impose. Il le conçoit donc comme simple différence entre l'avant et l'après, comme événement, comme contrat de société.

Comme il est naïf de venir alors objecter que la théorie est mal construite dans son ensemble. Qu'il ne faut pas commencer par les droits, mais par les devoirs, car c'est la seule manière d'arriver, avec la justification du droit, à une dichotomie nette entre droit et non-droit ${ }^{23}$. Cela provoque l'effondrement de toute la construction complexe de la dé-paradoxalisation du droit. On ne peut, maintenant, faire autrement que supposer que le droit est le contraire du non-droit, et que l'autre a un droit à ce que l'on remplisse les obligations correspondantes. La justification du droit est désormais formulable sans lacunes, mais aussi sans conscience du problème. Elle est aplanie de telle sorte que la pratique du droit sait à quoi elle peut s'en tenir et ne court pas le risque de perpétrer par mégarde le non-droit en poursuivant le droit.

Pour illustrer ce qui s'effondre ici, reprenons encore une fois le processus de déparadoxalisation. Le paradoxe est formulé en ces termes : "Droit parce que non-droit » (ou encore : «non-droit parce que droit»). Ce dont il résulte dans un premier temps, à l'état de nature : «droit est non-droit » (ou encore : non-droit est droit). La distinction est encore inintelligible, parce qu'il n'existe pas encore de devoirs. Du fait de l'introduction de la loi naturelle, cela devient : «droit n'est pas non-droit » (ou encore : «non-droit n'est pas droit»). On peut maintenant travailler avec logique, avec le principe de l'interdiction de la contradiction et du tiers exclu (pour arriver ainsi chez É piménide ou Gödel). La référence à soi-même est désormais elle aussi interdite, et avec elle la distinction de la distinction. Le système se déconnecte ainsi de l'observation de soi. Et pour finir, la consigne opératoire qui diversifie le système est la suivante : «les droits de l'un sont les devoirs de l'autre». La transformation parcourt donc ces différents paliers : «A parce que non-A », "A est non-A », "A n'est pas non-A » et "A est $B$ ». Dans la dernière forme, le paradoxe n'est plus donné que comme flou, et le juriste doté de compétences techniques en vient à bout.

\section{V.}

Ce que Jeremy Taylor admet pour la législation naturelle donnée par Dieu vaut aussi pour d'autres modes de dé-paradoxalisation. Ils tiennent compte des situations et des circonstances historiques dans lesquelles ils accomplissent leur ouvrage et doivent chiffrer leurs petites tricheries. Pour cerner, d'un point de vue historique, l'invention des natural rights, il sera par conséquent utile de se demander comment leur problème a été résolu avant et après.

Mais quel problème? Dans un autre texte, j'ai suivi cette question sous la prémisse d'une similitude sémantique profonde du ius, des natural rights et des droits subjectifs utilisés jusqu'à ce jour. Il en ressortait, à titre de tendance, un gain en abstraction dû au 
fait que la compensation des droits par les devoirs et par là-même toutes les exigences d'équité avaient été soustraites au droit de l'individu et déportées, par un détour, sur l'ordre juridique dans son ensemble ${ }^{24}$. Toutefois, en prenant un autre problème comme point de départ, on tire de l'histoire de tout autres fils de continuité et de discontinuité. Nous nous demandions comment l'impossibilité de trancher dans la question du droit et du non droit, impossibilité qui est constitutive du droit, est traitée dans le droit luimême. Ce thème a depuis toujours été traité, et il l'est encore, sous les concepts d' abrogatio et de derogatio. Et il se rencontre à partir du XVIII ${ }^{e}$ siècle dans la thèse qui fait de la violence l'origine du droit. L'époque qui nous intéresse, les XVII ${ }^{e}$ et XVIII ${ }^{e}$ siècles, applique manifestement tout un éventail de concepts, certains chargés de tradition, d'autres non, à ce problème - comme si on l'avait ressenti avec une urgence toute particulière dans la transition vers la société moderne et à l'époque des théodicées.

Dès l'Antiquité, on avait discuté de l'epieikeia/aequitas comme d'une institutionréceptacle pour le droit inique. Le problème fut ainsi pourvu d'un nom spécifique, et du même coup englouti. La judiciarisation croissante des relations sociales au Moyen Âge avait produit une autre casuistique sous le point de vue de la rupture du droit (abrogatio/derogatio) - par exemple : vol par nécessité, mais aussi droit de résistance ou droits particuliers du prince. Le débat particulièrement animé sur la raison d'État avait ainsi été repris en main ${ }^{25}$ ? Le paradoxe était dès lors nourri sur les plans théologique et juridique. Il apparaissait dans une série de types de cas connus et de concepts traditionnels, un peu en dehors de l'ordre, mais capables de s'y rattacher. Dans le Ductor Dubitantium, ce matériau apparaît comme le dernier chapitre du livre consacré aux Human Laws, c'est-à-dire déjà en dehors (ou en dessous) du droit naturel ${ }^{26}$. Le chapitre commence avec "equity» et s'achève avec "abrogation». Entre les deux, on traite entre autres de la «judicial interpretation», comme s'il s'attachait encore au traitement d'indéterminations immanentes au droit un reste de cette mauvaise réputation née $\mathrm{du}$ fait que le droit, dans son ensemble, ne fonctionne pas de manière tout à fait satisfaisante. Mais dans le contexte des human laws, toute la chaîne de problèmes se trouve à ladisposition du « supreme power » qui, en 1660, venait tout juste de s'établir. C'est manifestement cette "disposition» traditionnelle de la dogmatique $\mathrm{du}$ droit qui laisse insatisfait et qui suggère de laisser dormir ici le problème de l'incomplétude logique de l'ordre de droit pour le reprendre par d'autres moyens au sein du droit naturel.

Les anciennes versions du problèmes sont transmises sous la forme à laquelle l'on s'est depuis longtemps habitué. On les trouve chez Kant dans une systématisation particulièrement élégante, et on les discerne aussi dans leur irrémédiable équivoque, à la fin de l'État absolu. Dans l'« Appendice à l'introduction à la doctrine du droit » de la Métaphysique des Mcurs, c'est-à-dire en marge de la systématique, se trouve la théorie $\mathrm{du}$ droit équivoque (ius aequivocum); l'équité, comme droit sans contrainte, et le droit de nécessité, comme contrainte sans droit, sont proprement intégrés l'un à l'autre, comme s'il s'agissait de chiffrer le paradoxe en les représentant comme des jumeaux, et même comme un «symbolon » au sens originel de ce terme ${ }^{27}$.

La manière dont Kant se sort du problème n'est compréhensible que si l'on voit qu'est apparu entre-temps encore un autre lieu pour le conserver : la contrainte ou la force. Une plus forte accentuation de la contrainte comme base de validité du droit naturel semble s'être lentement imposée, couplée à un déplacement de la sémantique de la Gewalt, depuis la potestas vers la potentia et de là à la vis, puissance, force ${ }^{28}$. On peut 
considérer que l'expérience de l'absolutisme est ici décisive, mais il s'y ajoute le renoncement à un continuum de la rationalité, semblable à celui défendu en dernier lieu par les néo-stoïciens, comme nous l'avons vu plus haut. La raison ne peut plus être postulée à la fois comme base de la validité et de la connaissance. Et qu'est-ce qui peut alors justifier la validité du droit naturel, sinon le fait qu'il soit pourvu de possibilités de contrainte (que l'on peut tout à fait considérer comme "rationnelles») ? Cela devient au XVIII ${ }^{\mathrm{e}}$ siècle la conception dominante.

Une fois cela assuré apparaît une nouvelle possibilité pour localiser le non-droit du droit dans le droit. Il s'agit de la Gewalt. Dans la question de l'origine du droit, on devient ainsi indépendant aussi bien de la théologie que des spéculations sur un état de nature. Ainsi disparaissent également les constructions contractualistes qui n'avaient pas pu éviter de présupposer leur propre capacité à établir des liens. Tout est désormais beaucoup plus simple : l'origine du droit est un principe étranger au droit : la Gewalt ${ }^{29}$ la force ou la puissance. Il s'avère ainsi, dans le même temps, que c'est la société qui justifie le droit, et non pas le droit qui justifie la société, que la véritable garantie de l'ordre réside dans la propriété (laquelle sera interprétée comme Gewalt par ceux que l'on appellera plus tard les «socialistes ») et que le droit n'a justement pas besoin de justification supplémentaire comme instrument de cet ordre. Ou bien l'on peut supposer, avec Kant, que toute Gewalt, même si elle est au début dénuée de droits, est habitée par une téléologie débouchant sur la constitution du droit, et qu'ainsi, au fil de l'histoire, elle se porte d'elle-même à la forme du droit ${ }^{30}$.

Dans ce contexte, le non-droit du droit peut désormais se présenter de nouveau, sous forme de Gewalt dressée contre la Gewalt établie, de violence contre la force ${ }^{31}$ (Sorel) ou de critique de la Gewalt (Benjamin). Le problème atteint ainsi d'un côté une radicalité inutilisable qui peut s'affaiblir jusqu'à devenir un mouvement de sympathie avec les manifestants et non avec la police; mais il acquiert aussi, d'un autre côté, une pertinence surprenante dans la théorie du droit si l'on conçoit désormais la violence comme une décision prise en dépit $d u$ " caractère indécidable, en dernière instance, de tous les problèmes de droit ${ }^{32}$ ", c'est-à-dire comme corrélat de l'incomplétude logique de ordre juridique, c'est-à-dire comme flou et ambiguïté du droit ${ }^{33}$, et qu'on la remet ainsi à nouveau, assortie d'une réserve générale de caractère sociologique, aux mains des juristes. De loin, on se rappellera une fois encore Jeremy Taylor, qui avait traité de la judicial interpretation précisément là où il était question d'equity et d'abrogation, de droits coutumiers contraires au droit, de frontières du rationnel et d'autres impuretés dans le droit ${ }^{34}$.

\section{VI.}

Comme on peut le déduire de cette brève esquisse des thèmes liés à notreproblématique, les natural rights n'ont eu qu'une signification transitoire pour le problème du paradoxe logique du droit. Ils ont été très rapidement normalisés dans le passage vers un ordre social plus fortement marqué par l'individualisme, ils ont recruté sinon des devoirs, du moins des reconnaissances qui leur correspondent, et ils sont alors traités comme des droits qui existent d'ores et déjà (ce dont on peut aussi trouver la trace écrite dans les constitutions). Ils ont abandonné leur référence au problème, ils ne lui doivent plus qu'une certaine primordialité que l'on exprime aujourd'hui dans la dogmatique du droit à travers la référence aux droits fondamentaux. L'ambivalence du 
droit ne peut plus être fixée en partant de l'homme, elle est redevenue, comme jadis, l'affaire du droit lui-même, cela non plus désormais avec un arrière-plan théologique, mais sociologique: en tant qu'expression ambivalente d'une société qui ne semble justement pas faite pour rendre l'homme parfait ni même seulement heureux.

Que l'on ait pu considérer la chose autrement durant une brève période, même si ce fut toujours au prix de contradictions, donne à réfléchir. Si l'on veut s'engager dans une interprétation risquée, on pourrait supposer que la sémantique sociale a dû, pour la brève période d'une mutation sociale radicale, s'appuyer sur l'homme, parce que rien d'autre n'offrait de garanties suffisantes; et que l'on a dessiné cet homme avec des traits si négatifs, si égocentriques, si turbulent et si ambitieux, comme pour indiquer qu'il restait à développer un ordre qui fût adapté à tout cela ${ }^{35}$. Ensuite, il a justement fallu marquer alors, fût-ce aux dépens de toute plausibilité empirique, ces moments qui faisaient apparaître le besoin d'ordre au lieu d'admettre l'ordre comme déjà existant, comme donné avec la nature raisonnable et sociale de l'homme.

Si l'on veut clarifier les conditions de possibilité de cette innovation dans la perspective de la théorie de l'évolution, il faut avant tout dissocier la question de la variation du patrimoine intellectuel traditionnel de la question de la sélection. Dans les positions de départ existantes, le volontarisme théologique offrait, lorsqu'il rencontrait d'autres questions de fond, un potentiel de variation particulier dont les effets étaient normalement inhibés dans la mesure où il suffisait d'y réfléchir pour les considérer comme absurdes et de pas les pousser pas plus avant, mais qui, dans des conditions particulières, pouvaient tout de même subitement paraître utilisables. La réduction de toute la nature, qui apparaît grosso modo si constante, à un corrélat contingent de la volonté créatrice de Dieu, est l'une de ces invraisemblances. Le fait que notre propre bonne volonté et la qualité morale de notre manière de vivre ne soient pas déterminantes pour le salut de notre âme était un autre exemple ${ }^{36}$. L'idée d'un droit naturel (et ce en tant que "right», à la différence des qualités naturelles ou des lois naturelles de l'espèce humaine, que l'on a toujours considérées avec beaucoup de scepticisme) sans aucun corrélat social, était tout aussi absurde et a donc aussitôt suscité des répliques qui n'eurent aucune peine à démontrer qu'on n'appréhendait pas par là de façon pertinente l'être humain. Il a fallu une condition particulière, relevant de la situation historique disponible, pour permettre de briser ces barrages de plausibilité et la proposer à la réflexion.

J'avais déjà indiqué une explication facile à cette plausibilité temporaire: avec le radicalisme conceptuel des natural rights, on pouvait répondre au radicalisme politique des natural rights, on pouvait tenir compte des objections répandues, et que les troubles de la guerre civile n'avaient pas précisément limitées, contre les prétentions illimitées à l'égalité et à la liberté considérées comme des droits naturels, en reconnaissant les droits et en leur retirant leur légitimation. L'innovation se déplaça de la pratique politique vers la théorie où elle fut traitée et dans le même temps le progrès fut dévié dans une autre direction politique ${ }^{37}$. Hobbes et Taylor étaient des royalistes.

Une autre condition de plausibilité, exerçant un effet plus indirect, tenait cependant aussi aux transformations des conditions économiques réelles et de la théorie de l'économie. L'économie réelle avait, bien avant « l'industrialisation », connu un profond processus de transformation et elle s'était peu à peu centrée sur les opérations utilisant le truchement de l'argent. La théorie économique avait suivi ce processus : au XVII ${ }^{\mathrm{e}}$ siècle, en particulier (mais beaucoup moins au cours de la première moitié $\mathrm{du}$ XVIII ${ }^{\mathrm{e} 8}$ ) 
elle a attiré l'attention sur les liens, réglés par des lois naturelles, entre des faits économiques. ${ }^{39}$ Des faits économiques comme les hausses de prix et les difficultés d'écoulement des marchandises, le chômage et le manque d'exploitation des capacités productives (en dépit de la propriété individuelle!), les évolutions du marché international, mais aussi et notamment les succès spectaculaires des Hollandais, ne se laissaient plus décrire selon un schéma de vertu, comme une conséquence du labeur et du savoir-faire, ou de leur contraire, et même plus comme dépendant de la qualité des marchandises que l'on produit. Ils n'étaient manifestement pas le résultat de qualités saisissables en termes moraux, on ne pouvait donc pas non plus les concevoir comme une attention spéciale et préalable de Dieu (providentia) ou comme une récompense accordée par Dieu pour un comportement qui lui ait plu. Il fallait les attribuer aux lois spécifiques de l'économie, comme un effet du caractère indirect du mode d'action de l'argent, si toute peine ne devait pas être vaine. Dans le même temps, ces mêmes conditions économiques, justement, libéraient une quête sans fin pour obtenir plus d'argent. Plus de foin et plus de serviteurs, plus de pièces dans le château ou plus d'argenterie dans le buffet - tout cela avait des limites naturelles, même si l'un copiait les besoins de l'autre. Avec l'argent, cette quête trouva un objet sans limite et devint ainsi elle-même sans limite et insatiable. L'argent abolit en particulier toutes les barrières sociales posées à l'imitation des besoins et des efforts. Pour ce qui concerne l'argent, chacun pouvait copier chacun, les classes supérieures pouvaient même copier les classes inférieures, la noblesse le bourgeois ${ }^{40}$. Dans ce contexte, on ne pouvait pas repousser sans autre forme de procès, en les considérant comme irréalistes, le nouveau concept des natural rights et la différence de niveau qu'il suppose entre la quête individuelle et l'ordre de la loi naturelle. On peut cependant douter qu'il ait été donné au théologien et au philosophe de voir la situation économique sous cet angle. Il était toujours beaucoup plus tentant de juger l'avidité et l'insatiabilité des riches à l'ancienne manière, ou de les évaluer positivement, dans une mise en paradoxe directe, comme facteur de production et comme " publick benefit $»^{41}$. Mais l'économie elle-même avait déjà produit sa propre théorie réflexive et l'on ne pouvait plus ignorer l'anthropologie qui allait de pair avec elle.

Le concept des natural rights présenté ici a profité de ces chances exceptionnelles de retenir l'attention et d'être connecté, du moins le contexte d'une controverse théorique. Mais la plausibilité, à elle seule, ne garantit pas encore la possibilité d'utiliser le concept de manière quotidienne; et la sélection par l'évolution, au moins dans cette phase tardive de l'évolution sociale, ne signifie pas encore d'emblée la stabilisation de l'innovation. Les «natural rights» d'un Hobbes ou d'un Taylor étaient bien trop proches pour cela du paradoxe créateur de droit, à une proximité dangereuse de ce «trou noir " qui engloutit tout et ne laisse échapper aucune information sur soimême, mais est uniquement reconnaissable à un certain désordre autour de lui. Car les droits naturels perdirent aussi très vite leur caractère paradoxal, ils furent normalisés dans le sens de droits reconnus ou qui exigent et imposent politiquement leur reconnaissance. Ce qui a été conservé, comme gain d'abstraction, est le fait que chaque droit subjectif individuel ne devait pas forcément être payé au prix d'un devoir lié au même état de fait. Dans ce sens tout à fait concret, on ne peut plus désormais parler d'« équité » au sein des différentes institutions du droit. La différence de niveau visée dans la distinction "natural rights / natural law», qui est aussi utilisable comme instrument de séparation logique, a été transformée en nouvelles représentations de l'ordre, notamment économiques. Une société qui accorde des droits subjectifs et 
admet même qu'ils soient mis en forme par voie contractuelle est confrontée aux conséquences de ce déséquilibre. Et si la législation naturelle de Dieu ou sa «invisible hand » ne suffisent plus, on adresse les exigences correspondantes au système politique. Raison pour laquelle il devient plus convainquant de formuler le caractère paradoxal du droit en tant que Gewalt, et non plus comme un droit naturel sans droit.

\section{BIBLIOGRAPHIE}

Appleby, J. (1976) : «Ideology and Theory : The Tension between Political and Economic Liberalism in Seventeenth Century England », in : American Historical Review,81 (3), p. 499-515.

Appleby, J. O. (1978) : Economic thought and Ideology in Seventeenth-Century England, Princeton, Princeton University Press.

Benjamin, W. (1974) : «Pour une critique de la violence ", in : id. : L'homme, le langage et la culture, trad. par M. de Gandillac, Paris, Denoël, p. 23-55.

Borges, J. L. (1972 [1956]) : «El jardin de senderos que se bifurcan », in : id. : Ficciones, Madrid et al ., Alianza.

Brunner, O. / Conze, W. / Kosellek, R. (éd.) (1982) : Geschichtliche Grundbegriffe : Historisches Lexikon zur politisch-sozialen Sprache in Deutschland, vol. 3, Stuttgart, Klett-Cotta.

Cumberland, R. (1672) : De legibus naturae disquisitio philosophica, Londres, Hooke.

de Mattei, R. (1953) : « Il problema della Deroga e la "Ragion di Stato" », in : Castelli, E. (éd.) : Cristianesimo e Ragion di Stato, Rome / Milan, Bocca, p. 49-60.

Dumouchel, P. / Dupuy, J.-P. (1979) : L'enfer des choses. René Girard et la logique de l'économie, Paris, Seuil.

Folkers, H. (1979) : « Zum Begriff der Gewalt bei Kant und bei Benjamin », in : Figal, G. / Folkers, H. (éd.) : Zur Theorie der Gewalt und Gewaltlosigkeit bei Walter Benjamin, Heidelberg, FEST, p. 25-57.

Fries, J. F. (1803) : Philosophische Rechtslehre und Kritik aller positiven Gesetzgebung, Iéna, Mauke. Grotius, H. (1720) : De iure belli ac pacis,Amsterdam, Jansson Waesbergius.

Hofstadter, D. R. (1979) : Gödel, Escher, Bach: An Eternal Golden Braid, Hassocks, Harvester Press.

Holmes, S. (1985) : « Differenzierung und Arbeitsteilung im Denken des Liberalismus », in : Luhmann, N. (éd.) : Soziale Differenzierung: Zur Geschichte einer Idee, Opladen, Westdeutscher Verlag, p. 9-41.

Hooker, R. (1954) : Of the Laws of Ecclesiastical Polity, Londres, Dent.

Hoopes, R. (1950) : «Voluntarism in Jeremy Taylor and the Platonic Tradition », in : The Huntington Library Quarterly,13, p. 341-354.

Linguet, S.-N. H. (1767) : Théorie des loix civiles, ou Principes fondamentaux de la société, 2 vol., Londres, s. n. 
Luhmann, N. (1980) : «Frühzeitliche Anthropologie : Theorietechnische Lösungen für ein Evolutionsproblem der Gesellschaft », in : id. : Gesellschaftsstruktur und Semantik, vol. 1, Francfortsur-le-Main, Suhrkamp, p. 162-234.

Luhmann, N. (1981) : « Subjektive Rechte : Zum Umbau des Rechtsbewußtseins für die moderne Gesellschaft », in : id. : Gesellschaftsstruktur und Semantik, vol. 2, Francfort-sur-le-Main, Suhrkamp, p. 45-104.

Mandeville, B. (1924 [1714]) : The Fable of the Bees: or Private Vices, Publick benefits, Oxford, Clarendon Press.

Monk, S. H. (1960 [1935]) : The Sublime: A Study of Critical Theories in XVIIIth-Century England, Ann Arbor, University of Michigan Press.

Pufendorf, S. (1735 [1673]) : De officio hominis et civis iuxta legem naturalem libri duo, Cambridge, Thurlbourn.

Pufendorf, S.(1744 [1672]) : De iure naturae et gentium libri octo, Francfort-sur-le-Main / Leipzig, Knoch.

Resta, E. (1984) : L'ambiguo diritto, Milan, Angeli.

Spencer Brown, G. (1972 [1969]) : Laws of Form, New York, Julian Press.

Taylor, J. (1851-1852) : Ductor Dubitantium, in : id. : The Whole Works,éd. par R. Heber, vol. IX et X, Londres (réimpr. Hildesheim, Olms, 1970).

Varela, F. (1975) : « A Calculus for Self-reference », in : International Journal of General Systems, 2, p. 5-24.

Villey, M. (1964) : « La genèse du droit subjectif chez Guillaume d'Ockham », in : Archives de philosophie du droit,9, p. 97-127.

Villey, M. (1969) : « Le droit de l'individu chez Hobbes », in : Koselleck, R. / Schnur, R. (éd.) : Hobbes-Forschungen, Berlin, Duncker \& Humblot, p. 173-197.

\section{NOTES}

1. Cf. Benjamin (1974).

2. Pour beaucoup d'éléments, cf. Fries (1803), en particulier p. 24 sqq.

3. Cf. Spencer Brown (1972 [1969]). Voir cependant aussi sur ce point la prolongation du calcul à laquelle se livre Francisco Varela (1975). Ses réflexions autour d'une théorie des systèmes autoréférentiels, qui prévoient la self-indication comme une opération distinguant le système dans le système, en sont encore aux commencements. Mais elles fournissent déjà un motif de considérer avec un peu plus de distance les traditions de pensée qui se fondaient sur une interdiction de l'autoréférence.

4. Ce que l'on voit avec une clarté singulière dans Borges (1972 [1956]).

5. Cf. Taylor (1851-1852).

6. Ibid., p. 280.

7. Que l'on considère avant tout le changement de direction que subit le point centralde l'argumentation. Pour ce qui concerne cet état de fait que constitue l'égocentrisme, et en se référant à la diversité des mœurs et à la fréquente transformation des lois, Carneades avait nié la validité du droit naturel. On y avait toujours opposé, jusque là, la socialité naturelle de l'homme. Ce que fit par exemple Hugo Grotius dans les impressionnants prolégomènes à De iure belli ac pacis,n $n^{\circ}$ 5-9 (cités d'après Grotius [1720], p. IV sq.). Ce qui était en jeu dans cette controverse était 
la conception de l'homme dont il fallait partir : d'une interprétation plutôt rapportée à soi-même ou plutôt sociale. Hobbes et Taylor sortent de ce débat en découvrant une troisième possibilité (qui n'a cependant rien d'un « compromis »). La théorie de l'égocentrisme est exacte et mérite malgré tout le titre de «droit ». Depuis le milieu du XvII ${ }^{\mathrm{e}}$ siècle, ici et ailleurs, des constructions théoriques plus risquées, d'un point de vue moral, sont aussi possibles.

8. Cf. Taylor (1851-1852), p. 283.

9. Ibid., p. 293.

10. Ibid., p. 295 : « For when God made a free agent, He by nature gave him power to do all he could desire $»$.

11. Cf. Hoopes (1950).

12. Taylor (1851-1852), p. 304

13. Ibid., p. 306.

14. Du point de vue interne au système, la théologie a vite fait de présenter une réponse toute prête : un homme qui penserait ainsi ne ferait selon lui que s'empêtrer dans les contradictions. Cf. Grotius (1720), 1.1. § X, 5, p. 12 : "Quanquam enim immensa est Dei potentia, dici tamen quaedam possunt esse, ad quae se illa non extendit; quia quae ita dicuntur, dicuntur tamen, sensum autem qui rem exprimat nullum habent, sed sibi ipsis repugnant".

15. Cf. Taylor (1851-1852), p. 304 : « This right of nature being now almost wholly taken from us, part of it is taken up to God, and part of it is deposited in the hands of the civil power, but we have none of it. "

16. On a toujours senti, sur ce point, la nécessité d'une argumentation. La citation de Grotius que nous venons de donner, visant à démontrer que la limitation de la toute puissance de Dieu n'est qu'apparente, se réfère à ce problème.

17. Taylor (1851-1852), p. 282 sqq. Un problème secondaire traité à cette occasion est intéressant pour les libertés acquises par ce biais. On sait que les animaux cessent de copuler après la conception. Les Pères de l'Église (Jérôme, Ambroise, Origène, Sedulius), en auraient conclu que selon le droit naturel, il était aussi interdit à l'homme d'avoir des relations sexuelles avec une femme enceinte. Or il s'agirait d'une interprétation très dangereuse, parce qu'elle pousserait les hommes mariés à commettre l'adultère. Chez les animaux, cette question n'est en aucun cas réglée par le droit naturel. (Beasts are indeed so ordered by nature, but without a law). Dans le cas des rapports humains, il faudrait pour cela une loi correspondant à la nature de l'homme.

18. Ibid., p. 283.

19. Il existe aussi, bien entendu, d'autres formes pour cela. Chez Richard Hooker, par exemple, on lit: " God... is a law both to himself, and to all other things besides " (Hooker [1954], I, II, 3, p. 152). La validité indéfectible du droit naturel peut ensuite être déduite du lien que Dieu s'impose à luimême.

20. Ainsi Holmes (1985).

21. Cf. Villey (1964); Villey (1969).

22. Cf. Hofstadter (1979).

23. C'est ce que Pufendorf objecte à Hobbes, puis de nouveau Fries à Kant. Cf. Pufendorf (1735 [1673]), I.I, chapitre II, § I-V, p. 51 sq. ; Pufendorf (1744 [1672]), notamment I.I, chapitre VI, § IVVI, p. 89 sq ; Fries (1803).

Par rapport à Hobbes ou Taylor, Pufendorf entame la théorie au deuxième niveau, parce qu'il ne peut (à fort juste titre) que voir de la confusion au premier. Cf. Pufendorf (1735 [1673]), p. 51 : « Alias enim si in tanta volutatis libertas, tantaque inclinationum \& studiorum diversitate, quisque citra reflexionem ad certam normam ageret, quod ipsi in mentem veniret; non poterat non maxima confusio in genere humano existere ».Tout comme chez Parsons, donc: sans orientation normative, pas de possibilité d'action! Cela est très plausible; seulement la théorie des natural rights n'était pas tenue d'affirmer la possibilité de mettre en application un état naturel au premier niveau. Elle devait et pouvait ignorer le temps.

24. Cf. Luhmann (1981). 
25. Cf. de Mattei (1953).

26. Livre III, chapitre VI (vol. X, p. 501 sq.) ; Le point de vue de la systématisation est « ways of the changing of human laws ".

27. Chez Kant, on ne trouve toutefois que l'explication moins profonde, voire totalement insatisfaisante, selon laquelle le problème est issu d'une "confusion » des motifs objectifs et subjectifs de l'exercice du droit, en raison de laquelle on suppose commeadmisel'existence de cas où le droit est douteux et qu'aucun juge ne peut être chargé de trancher. Et pourquoi pas ? C'était cela, la question!

28. [Les deux derniers mots en français dans le texte (N.d.T.).] Cf. les contributions à l'article «Macht, Gewalt » in : Brunner et al. (1982).

29. Cf., en termes polémiques contre les constructions du droit naturel et du contrat, Linguet (1767), vol. 1, p. 284 sq.

30. Cf. Folkers (1979).

31. Les deux termes en français dans le texte (N.d.T.).

32. Terme employé par Walter Benjamin (Benjamin [1974], p. 196).

33. Cf. Resta (1984), se rattachant à Benjamin.

34. Taylor (1851-1852), vol. X, p. 513 sq.

35. Pour d'autres points de repère, voir Niklas Luhmann (1980).

36. On le sait, c'est à ce point que s'est rattachée l'analyse de Max Weber. On pourrait la rajouter ici si l'on en remodelait les problématiques, passant de la causalité de la motivation à la théorie de l'évolution.

37. J'avais déjà fait allusion à Pufendorf. Cf. aussi Cumberland (1672). Hormis la tonalité sentimentale de l'élément social, qui deviendra bientôt une mode, cela n'apporte rien de nouveau, mais sert uniquement à remettre la nouveauté au niveau.

38. La re-moralisation flagrante de la théorie économique, qui débuta à la fin $d u x I_{I}{ }^{e}$ siècle, incita Mandeville à s'y opposer directement et a finalement fait apparaître Adam Smith comme le fondateur de l'économie nationale moderne, appellerait un traitement particulier. Elle pourrait confirmer la conception, défendue ici à propos des natural rights, selon laquelle la morale est le principal instrument de l'extinction des innovations relevant de l'évolution. C'est un fait propre à emporter la conviction, que l'on puisse, à l'aide du code moral, prouver que le bien est bon, et le mal mauvais, et que le monde est aménagé en conséquence. Les états de fait dont on ne peut répondre ainsi sont alors, justement en Angleterre, renvoyés au sublime, à l'épouvante, au romantisme du cimetière, au paradoxe, à l'humour et à l'ironie, mais aussi et notamment au diable. Sur ce point, cf. Monk (1960).

39. Cf. avant tout Appelby (1978), corrigeant certaines représentations du « mercantilisme ».

40. Cf. à ce propos, se rattachant à l'anthropologie du conflit d'imitation de René Girard, Dumouchel / Dupuy (1979).

41. Sur cette discussion, qui fut lancée très longtemps avant Mandeville, cf. les allusions dans l'introduction de F. B. Kaye à Mandeville (1924 [1714]); également Appleby (1976).

\section{INDEX}

Mots-clés : droits (naturels), droits (subjectifs), ordre

Schlüsselwörter : Rechte (natürliche), Rechte (subjektive), Ordnung 


\section{AUTEURS}

\section{NIKLAS LUHMANN}

Niklas Luhmann (1927-1998) était un des plus importants sociologues allemands de la deuxième moitié du vingtième siècle, fondateur de la théorie des systèmes. Pour plus d'informations, voir la notice suivante. 